\title{
Comments on "Inverse relationship between macular pigment optical density and axial length in Chinese subjects with myopia"
}

\author{
Xing-Ru Zhang $\cdot$ Zhen-Yong Zhang
}

Received: 28 January 2013 / Accepted: 26 February 2013 /Published online: 12 March 2013

(C) Springer-Verlag Berlin Heidelberg 2013

\section{Dear Editor:}

We read with interest the recent article by Tong [1] and would like to comment on it. The authors have conducted a study with a limited sample size and developed a plausible conclusion that macular pigment optical density is inversely associated with the axial length. This conclusion sounds reasonable and is consistent with the clinical observation that higher myopia seems to have less macular pigment. However, the weakness of this study in methodology should be considered when interpreting the findings.

Macular pigment optical density (MPOD) is believed to be a function of age, sex, body mass index, genetic susceptibility, and the levels of dietary intake of carotenoids that can modify the retinal levels of carotenoids [2]. This article has presented, among other things, that age confers no significant effect on MPOD based on a cross-sectional but not a longitudinal study. It is noteworthy that MPOD, also in a Chinese population, has been reported to tend to decline in a recent study [3], where measurement of MPOD is similar in methods to this study but with a bigger sample size of study population. The reasons for this discrepancy between these two studies, however, are not known; a well-matched group including a healthy study population could strengthen this study anyway. Also, other confounding factors have not been washed out with additional investigations in this study, among which the levels of dietary intake of carotenoids appear to be the foremost concern. Macular pigment is composed of three carotenoids [4]: lutein, zeaxanthin and meso-zeaxanthin, while lutein and zeaxanthin, neither of which can be synthesized by human body, are entirely of dietary origin. Accordingly, specification for whether the study subjects are routinely supplemented with lutein/ zeaxanthin is necessary.

To single out a potential factor for decline in MPOD, other confounding factors that may substantially affect the distribution and abundance of macular pigment should be considered to make a stronger conclusion.

Competing interests All authors have no conflict of interest.

\section{References}

1. Tong N, Zhang W, Zhang Z, Gong Y, Wooten B, Wu X (2012) Inverse relationship between macular pigment optical density and axial length in Chinese subjects with myopia. Graefes Arch Clin Exp Ophthalmol. doi:10.1007/s00417-012-2225-Z

2. Bone RA, Landrum JT, Guerra LH, Ruiz CA (2003) Lutein and zeaxanthin dietary supplements raise macular pigment density and serum concentrations of these carotenoids in humans. J Nutr 133 (4):992-998

3. Yu J, Johnson EJ, Shang F, Lim A, Zhou H, Cui L, Xu J, Snellingen T, Liu X, Wang N, Liu N (2012) Measurement of macular pigment optical density in a healthy Chinese population sample. Investig Ophthalmol Vis Sci 53(4):2106-2111

4. Bone RA, Landrum JT, Tarsis SL (1985) Preliminary identification of the human macular pigment. Vis Res 25(11):1531-1535
X.-R. Zhang

Liqun Hospital of Putuo District, Shanghai, China

X.-R. Zhang $\cdot$ Z.-Y. Zhang $(\bowtie)$

Department of Ophthalmology, Putuo Hospital, Shanghai

University of Chinese Traditional Medicine, No. 164,

Lanxi Road, Shanghai 200062, China

e-mail: zzyly818@sina.com 